\title{
Reference nanometrology based on AFM, SEM and TEM techniques
}

\author{
Gaoliang Dai, Wolfgang Häßler-Grohne, Jens Fluegge, Harald Bosse \\ Physikalisch-Technische Bundesanstalt, 38116 Braunschweig, Germany
}

\begin{abstract}
Highly accurate dimensional metrology of nano structures are increasingly demanded for quality assurance in various nanomanufacturing industries. This paper presents some recent progresses on the developments of reference nanometrology at PTB based on AFM, SEM and TEM techniques: (i) the enhancement of the measurement speed of PTB's Met. LR-AFM up to $1 \mathrm{~mm} / \mathrm{s}$ (i.e. $50 x$ faster than before), thus significantly increase its measurement throughput and reduced the measurement drift; (2) the development of the 3D/CD-AFM, which is capable of true 3D measurements of nanostructures either with flared AFM tips or in the tilting-AFM principle; (3) the realisation of a new bottom-up traceability approach which applies the ${ }^{28} \mathrm{Si}$ crystal lattice as internal "rulers" based on TEM; (4) CDSEM metrology with developed simulation software, a modular Monte Carlo software package MCSEM, for the simulation of electron signal contrast; and (5) the development of a new CD reference material referred as IVPS100-PTB.
\end{abstract}

Keywords: Nanometrology, traceability, calibration, atomic force microscopy (AFM), scanning electron microscopy (SEM), transmission electron microscopy (TEM), feature width, critical dimension (CD), sidewall angle (SWA), Line Edge Roughness (LER), Line Width Roughness (LWR), standards, Monte Carlo simulation.

\section{Introduction}

Only the things which we can measure, they can be investigated, understood, controlled and improved. Nanoscale metrology is a fundamental task of developing various nanotechnologies. For instance, in the semiconductor industry, progressive miniaturisation of advanced techniques such as the Extreme Ultraviolet (EUV) lithography, nanoimprint and Directed Self-Assembly (DSA) currently delivers nanodevices with feature size below $20 \mathrm{~nm}$. Accurate measurement of the dimensional parameters (including the critical dimension $C D$, height, sidewall angle, line-edge roughness(LER)/line-width roughness(LWR), etc. as shown in figure 1) of nanostructures both on silicon wafers and photomasks is a crucial task for process development and process control. As stated in the ITRS metrology roadmap [1], the measurement uncertainty of the physical CD needs to be reduced to $1.6 \mathrm{~nm}$ (year 2016) and $0.7 \mathrm{~nm}$ (year 2024). In the photonic industry, applying nanophotonic structures and devices promises reduction in energies operation, densely integrated information systems, enhanced spatial resolution for imaging and patterning, and new sensors for increased sensitivity and specificity [2]. However, the development and manufacture of different types of nanophotonic components e.g. diffractive optical elements, waveguides, Bragg gratings and nanophotonic interconnects requires traceable characterisation tools to ensure their proper functionality.

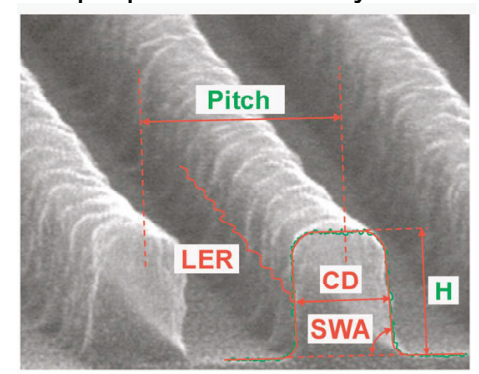

Fig.1 Dimensional parameters of nanostructures both on silicon wafers and photomasks needed to be accurately measured for process development and process control, shown as critical dimension (CD), height $(H)$, sidewall angle (SWA), line-edge roughness (LER) and pitch.

The aim of the reference nanometrology is to ensure the traceability and accuracy of various on- and off-line nano measurement tools such as optical scatterometry, atomic force microscopy (AFM) and scanning electron microscopy (SEM), thus to ensure reliable nanomanufacturing as illustrated in figure 2 . The metrological traceability is defined in the International vocabulary of Metrology (VIM) as 
"property of a measurement result whereby the result can be related to a reference through a documented unbroken chain of calibrations, each contributing to the measurement uncertainty" [3]. The lack of traceability in measurements inhibits the comparison of tools from different manufactures and limits knowledge about the real size of fabricated features [4].

In this paper, several new progresses on the developments of reference nanometrology at PTB based on AFM, SEM and TEM techniques will be presented.

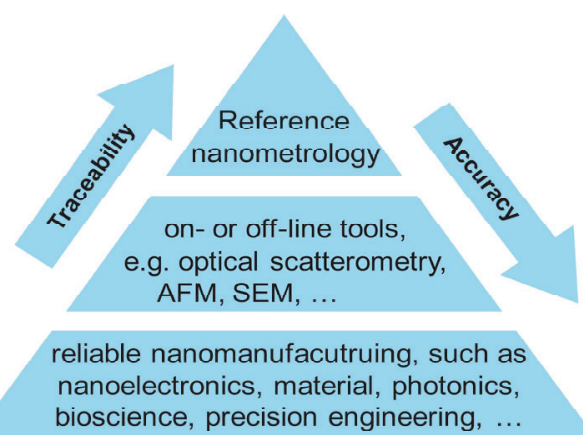

Fig. 2 The role of the reference nanometry in nanotechnology related industry.

High speed metrological large range AFM AFM technique has advantages such as high lateral and vertical resolution, (almost) nondestructive and (quasi) 3D measurement capabilities, becoming a kind of most widely used nano measuring tool today. Metrological AFMs have been developed in the past two decades [5-8]. Compared to commercial AFMs, they are typically equipped with laser interferometers for measuring the scanner's motion. In such a way, the displacement measurements performed by metrological AFMs are directly linked to the definition of the metre. Metrological AFMs have been widely used for reference nanometrology tasks, such as the calibration of step height standards, $1 \mathrm{D} / 2 \mathrm{D}$ gratings, and 3D form of nanoindenters.

Conventional AFMs usually have limited measurement range, typically tens of micrometres. To overcome this limit, several metrological large range AFMs (Met. LR-AFM) have been built up recently $[5,9]$. In these LRAFMs, a mechanical stage which has a motion range of several millimeters is combined with a piezo stage for scanning the sample. In such a way, surfaces can be measured over a large area with a speed of typically 10-50 um/s [5]. Unfortunately, till now the low measurement speed remains as a major shortcoming of LRAFMs. It leads not only to a low measurement throughput, but also to a significant measurement drift over the long measurement times needed (up to hours or even days), particularly for measurements over a large area.

The design of the high speed Met. LR-AFM is based on our previously developed Met.LRAFM [5]. The schematic diagram of the instrument is shown in Figure $3(a)$. In the instrument, the sample is fixed on a piezo stage, which is in turn mounted on the mirror corner of a high precision nano measuring and positioning machine (NMM) [9]. In this NMM, the mirror corner stage is moved by stacked mechanical $x-, y-$ and $z$-stages, which offer a motion range of $25 \mathrm{~mm}, 25 \mathrm{~mm}$ and $5 \mathrm{~mm}$, respectively. Three interferometers (only that of $x$ - and $z$-axes are shown in the figure) and two autocollimators (not shown in figure) measure the six degrees of freedom of the mirror corner stage with respect to the metrology frame. By servo controlling the position and angles of the mirror corner stage, the NMM can position with nanometre accuracy [9]. The measurement loop of the instrument is shown as the red dot lines.

The Met.LR-AFM measures in the so-called scanning sample principle, i.e. the sample to be measured is scanned while the AFM head is kept stationary.

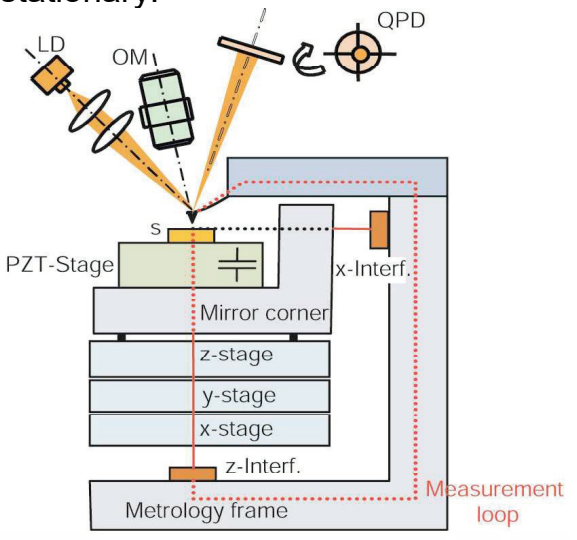

(a)

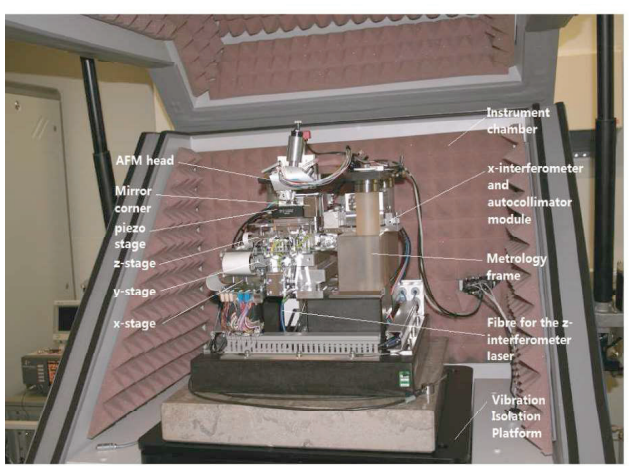

(b) 
Fig.3 (a) Schematic diagram of the high-speed metrological large range AFM (High speed Met. LR-AFM); (b) photo of the instrument

Several important design concepts have been implemented to realise both, high measurement speed and high metrology performance:

- the contact AFM mode is applied instead of the intermittent and non-contact modes, which offers shorter AFM response time and larger AFM sensing range.

- during measurements, the sample is scanned in the xy-plane solely by the NMM (such a motion usually has a constant velocity, therefore, high dynamics of the xyscanner is not needed.), however, a high dynamic $z$ motion of the sample is realised by a combined piezo stage and the z-sage of the NMM controlled in parallel.

- the AFM output signal is combined with the position readouts of the piezo stage and the NMM to derive the surface topography. The combination of these readouts offers a large bandwidth of measurement signals, thus provides high speed measurement capability.

- two important means are taken to reduce the distortion in measured profiles, namely (a) the time delay of sensor signals are corrected; (b) the position sensors of the AFM and piezo stage are traceably calibrated to the z-interferometer of the NMM in situ.

To demonstrate the metrology performance of the high speed Met.LR-AFM, figure 4(a) shows a same surface profile measured at different speeds (from $10 \mu \mathrm{m} / \mathrm{s}$ up to $500 \mu \mathrm{m} / \mathrm{s}$ ). It can be seen the quality of measured profile is very similar despite the scanning speed has been increased by 50 times.

AFM tip wear is a critical issue, particularly for high speed AFM. In this study, AFM tip type ContDLC (Nanosensor), whose tip is coated with a diamond like carbon (DLC) layer, has been applied for AFM measurements. The tip geometry is characterised by a tip characteriser (a kind of sharp silicon triangular nanostructure) before and after the measurements, as shown in figure 4(b), indicating very low tip wear.

For more design details and measurement results about the high speed Met. LR-AFM, the readers are referred to our paper [10].

\section{AFM for true 3D dimensional metrology of nanostructures}

Conventional AFM usually applies a conical or pyramidal shaped AFM tip measuring from the top. An image generated by an AFM is the dilated result of the real structure by the tip geometry. Consequently, it is not capable of true 3D measurements. For instance, the steep sidewalls and footing regions of nanostructures cannot be resolved, since the tip geometry prevents contact with those regions.

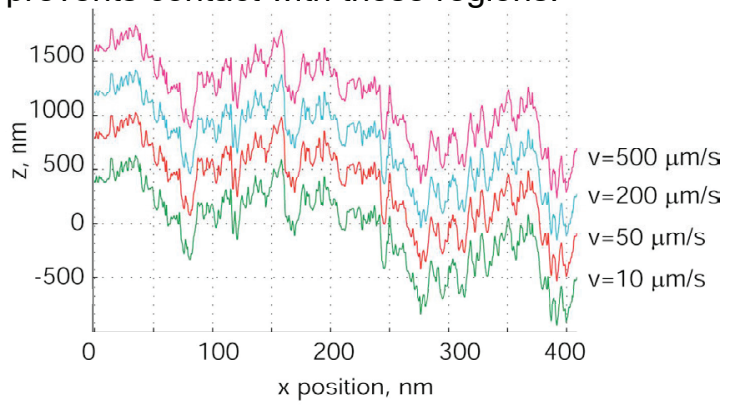

(a)

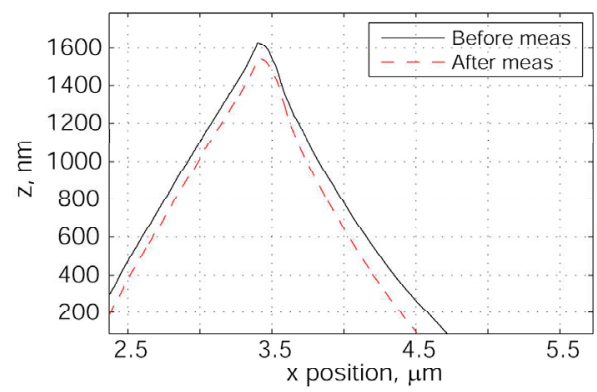

(b)

Fig.4 (a) Surface profiles taken on a PTB roughness standard (RN 505) by the high speed Met. LR-AFM, measured at the same location with different speeds from $10 \mu \mathrm{m} / \mathrm{s}$ up to $500 \mu \mathrm{m} / \mathrm{s}$; (b) Tip shape characterised before and after measurements. Profiles are intentionally shifted along the $y$-axis for clarity;

To achieve true 3D measurements of nanostructures, two different AFM techniques, the CD-AFM and the tilting-AFM, have been developed. The CD-AFM uses flared tips. Such tips have an extended geometry near its free end which enables the probing of steep and even undercut sidewalls, as shown in figure 5(a). The CD-AFM technique has advantages of measuring both the left and right sidewalls of nanostructures in only one measurement. However, it has disadvantages such as the relatively large tip geometry (typically tens to hundreds of nanometres) which limit its spatial resolution for measuring very dense structure patterns, and the complicated tip shape which makes the tip characterisation more difficult. measurement of dense structure patterns as well as for the corner rounding and footing of structures. But the tilting-AFM has its own limitations, too. In order to make the sidewalls of steep structures measurable, the AFM tip 
has to be tilted with respect to the structure by a certain angle, as shown in figure 5(b). As only one side of the structure is measurable at one tilted setup, either the AFM tip or the sample must be rotated so that the opposite side of the structure becomes measurable. Consequently, the images obtained at different tilting views must be stitched together to determine the $C D$, where the stitching error will strongly influence the measurement accuracy. Both techniques are realised in a 3D/CD-AFM developed at PTB [11], and are applied in a complementary manner, thus adding their strengths and overcoming their limitations.

The 3D/CD-AFM uses the classic optical lever technique to detect the bending and torsion of the cantilever. The AFM measurements are mostly performed in the intermittent-contact mode, where the amplitude modulation technique is applied for detecting the tip sample interaction. For achieving better CD measurement performance, new probing and measurement strategies have been developed. For instance, the tip is capable of probing surfaces with a vertical and/or a torsional oscillation to enhance the 3D probing sensitivity; a "vector approach probing" (VAP) method has been applied for enhancing the measurement flexibility and for reducing the tip wear as well. The tilting-AFM is an add-on function of the existing instrument, by applying a new type of AFM tips and manually tilting the scanner (together with the AFM head), while all other components including the controller and software are kept the same. The tilting angle is currently about 11 degrees in the tilting-AFM setup.

Two measurements using the 3D/CD-AFM are demonstrated in figure 6. Figure 6(a) shows an edge profile measured on an EUV photomask line feature using a super sharp AFM tip type SSS-NCLR (Nanosensor) using the tilting-AFM setup. Totally 32 repeated measurements are plotted with their details shown in the inset figure, showing excellent measurement repeatability. From such images, feature parameters such as SWA, corner rounding and footing can be determined. Figure 6(b) illustrates a measured 3D-AFM image of an IVPS 100 structure using a CDR-70-EBD tip (Nanosensor). After correcting the tip dilation effects with the calibrated tip geometry (detailed in the next section), the feature parameters such as CD, SWA, and LER/LWR can be obtained.

For more measurement and application examples of the 3D/CD-AFMs, the readers are referred to [11-12].

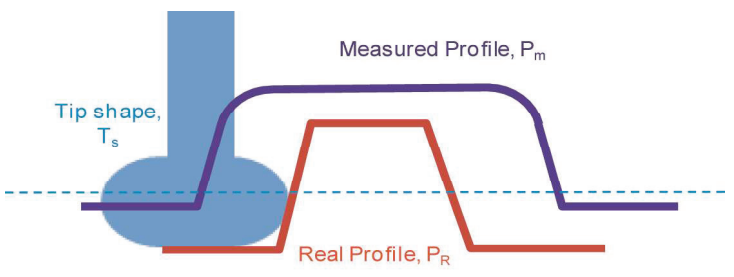

$\mathrm{P}_{\mathrm{m}}=\mathrm{PR}_{\mathrm{R}} \oplus \mathrm{T}$

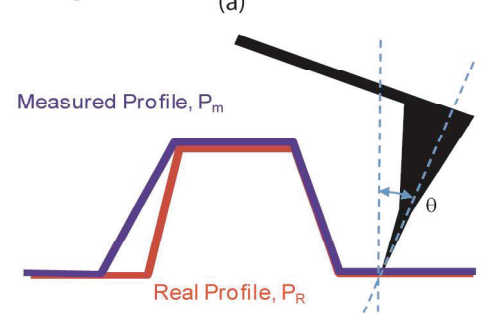

(b)

Fig.5 Principle of the CD-AFM (a) and tilting$A F M(b)$ for true $3 D$ measurements of nanostructures

\section{Bottom-up traceability approach by silicon crystal constant based on TEM}

Traceability is a fundamental issue for nano dimensional metrology. For realising traceable AFM 3D measurements, two important issues need to be met, the calibrations of the AFM's displacement scales and the effective tip geometry.

Thanks to the development of the state-of-theart metrological AFMs [5], currently the scaling factors of AFMs can be calibrated with a relative uncertainty of better than $10^{-3}$. Consequently this contribution to the overall measurement uncertainty of $3 \mathrm{D}$ metrology is typically insignificant, especially for the small feature sizes of the current technology node. Images produced by AFM measurement is a dilated result of the specimen feature by the effective geometry of the applied tip [13-14], as illustrated in Figure 7. Here, the phrase "effective geometry" means both, its physical tip geometry and the tip sample interaction treated as a simple geometrical expansion [9]. To retrieve the real feature's width from the measured AFM images, the effective tip width needs to be calibrated accurately and traceably, being a critical challenge today.

With the development of modern spherical aberration correction technique, currently the (scanning) transmission electron microscopes ((S)TEMs) are capable of microscopic imaging and microanalysis with a spatial resolution down to $0.05 \mathrm{~nm}$, i.e. true atomic resolution. Such outstanding imaging power offers new solutions for nanometrology. As illustrated in figure 8 , if the atoms in the nanostructures is resolvable, its dimensional parameters (shown as the line width) can be easily determined by using the atom spacing as an internal "ruler". A 
remarkable progress applying this idea is the development of SCCDRM (single crystal critical dimension reference material) by Dixson et al. [15]. Later Takamasu et al. [16] and our group [17] have also utilised this idea for traceable and high accurate line width metrology.

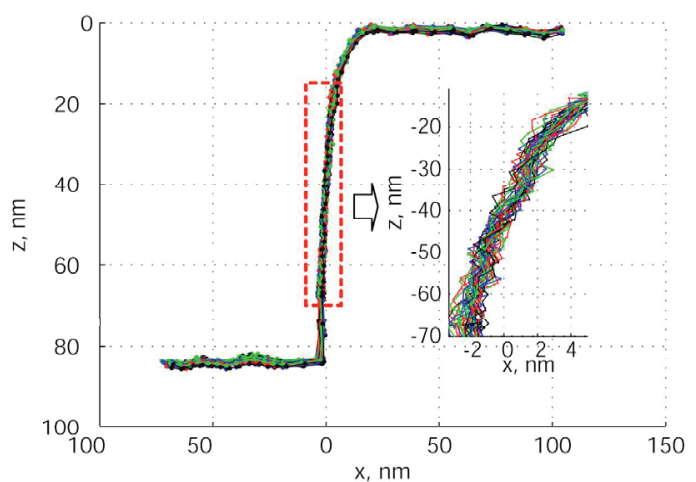

(a)

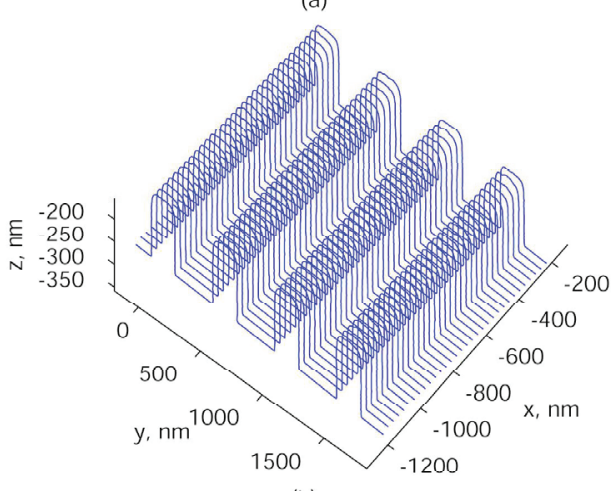

(b)

Fig. 6 (a) Edge profiles of an EUV photomask line feature measured by the tilting-AFM using a super sharp AFM tip type SSSNCLR. Totally 32 repeated measurements by the tilting-AFM are plotted, showing excellent measurement repeatability. The inset figure shows the zoom-in details of the sidewall at the marked area; (b) Measured 3D AFM image of an IVPS 100 structure by the $3 D-A F M$ using a CDR-70-EBD tip.

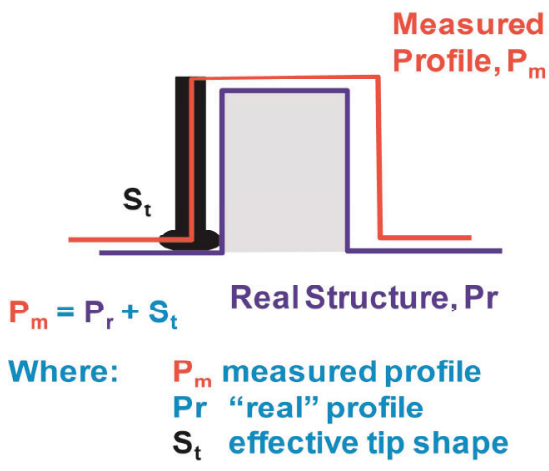

Fig.7 Schematic diagram showing the dilation effect of the AFM tip geometry. Consequently the measured $C D$ value is the width of the real structure offset by the effective tip width.

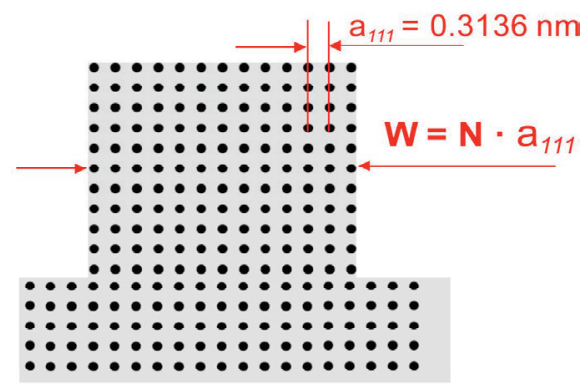

Fig.8 Bottom-up traceablility approach: determination of feature width using the crystal lattice constant as an internal "ruler".

A measurement example is presented in the Figure 9 to demonstrate the measurement power of the idea presented above. Before the measurement, a line feature made of crystal silicon is sample prepared using a dual-beam FIB instrument (Helios nanolab 660 of FEI) at the target measurement location. To protect the target feature not being influenced during the sample preparation, two protection layers (first a carbon layer followed by a platinum layer) are deposited to the target feature before the TEM lamella is prepared. Lamellas were produced utilizing the FIB lift out technique. The lamellas were further thinned to have a thickness of approx. $50 \mathrm{~nm}$ using $2 \mathrm{kV}$ $\mathrm{Ga}+$ ions before being measured in TEM.

Figure 9(a) shows the STEM image of the line feature measured in a high-end TEM device (Titan Themis 300 of FEI) at a magnification of $910 \mathrm{k}$ and with a resolution of $22.5 \mathrm{pm} / \mathrm{pixel}$. The structure at the top left corner in the marked box is zoomed in and shown in the figure 5(b). The crystal planes of $\left\{\begin{array}{lll}1 & 1 & 1\end{array}\right\}$ can be well resolved. Thus, according to the known value of atom spacing between the crystal planes $\left\{\begin{array}{lll}1 & 1 & 1\end{array}\right\}, a_{111}$, which was calculated traceably as $313.56011(17) \mathrm{pm}$ from the $d_{220}$ value determined by a combined $\mathrm{x}$-ray and optical interferometry [18], the width of the line feature is determined as $(44.3 \pm 0.3) \mathrm{nm}$. This result is also traceable to the $\mathrm{SI}$ unit of metre via an unbroken calibration chain.

\section{Reference nanometrology with SEM}

Today, scanning electron microscopy (SEM) is capable of high imaging resolution down to 1 $\mathrm{nm}$ even at relatively low energy level of about $1 \mathrm{keV}$. Compared to the AFM technique, SEM has higher measurement speed/throughput, being one of most widely used nanomeasuring 
tool in nanomanufacturing. For instance, for quantitative measurements of $C D$ and LER/LWR, specially adapted so-called CDSEM are used for quality control purposes in mask and wafer production.

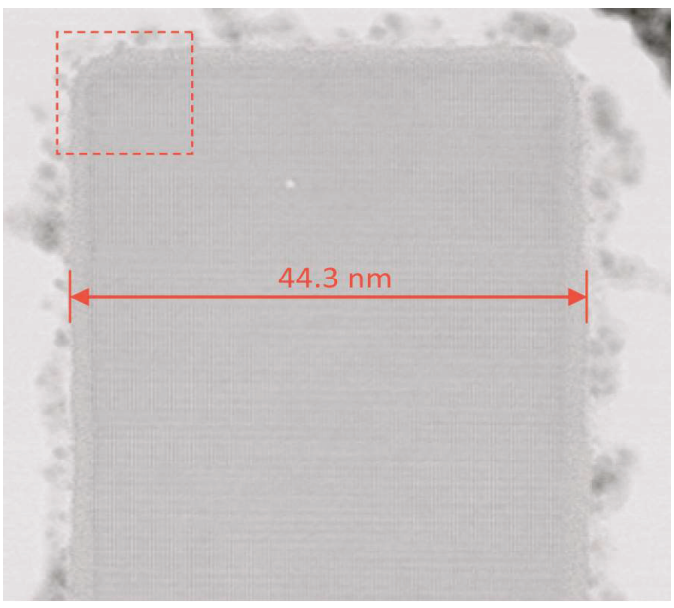

(a)

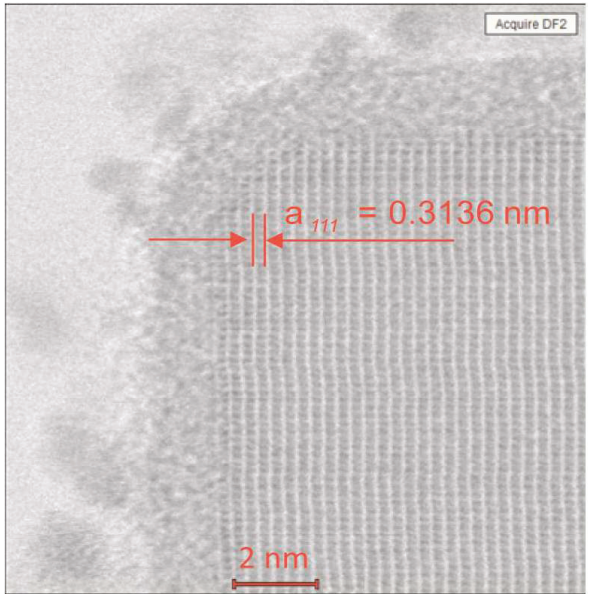

(b)

Fig.9 Width of a crystal silicon line feature determined by the bottom-up approach, shown as (a) the measured STEM image of the line feature; and (b) the zoom-in view of the image at the marked box in (a), where the crystal plane is clearly visible for being applied as an internal ruler.

Special care has to be taken in operation of the CD-SEM to achieve optimum reproducibility of the sample positioning and the imaging process. Figure 10 shows the so-called Electron-Optical Metrology System (EOMS) of the PTB, which combines a low-voltage SEM of the Zeiss ULTRA type and a large vacuum chamber which contains a laser-interferometer controlled 2D precision stage for positioning of larger planar substrates [19]. The magnification of the SEM can be calibrated traceably to the interferometer in-situ.

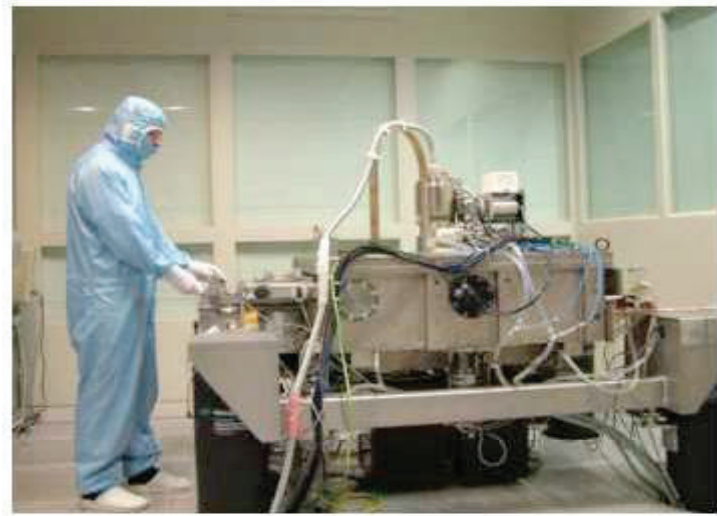

Fig.10 Photo of the Electron Optical Metrology System (EOMS) at the PTB. the ZEISS ULTRA SEM is mounted on top of the vacuum chamber

CD-SEM uses the secondary electrons (SE) generated within the sample surface as the measurement signal. To properly evaluate the feature parameters from the measured SEM image, one needs to apply a suitable physical model to investigate the interaction of the primary electrons with the material and the geometry of nanostructures. Different software program packages have been developed for this task. In order to simulate the stochastic nature of the different elastic and inelastic scattering events of the primary electrons as well as the resulting electrons (backscattered, secondary or transmitted electrons) in the solid stat, most of the software use Monte Carlo simulation method. An overview of the applied models is provided in [20], and a detailed description of MCSEM, the modular Monte Carlo software package for simulation of electron signal contrast developed at the PTB, has been published in [21]. Uncertainties of CD-SEM measurements at PTB for the width of high quality lithographic features of sizes down to $100 \mathrm{~nm}$ and below on photomasks and wafers as low as $U 95 \%=10 \mathrm{~nm}$ have been reached.

To verify the consistency of the 3D/CD-AFM and CD-SEM measurements, recently a comparison between both metrology tools in measuring the $C D$ of line features of a photomask standard has been carried out. The obtained CD results at $50 \%$ feature height for isolated opaque line features are plotted in figure 11, showing excellent agreement. 


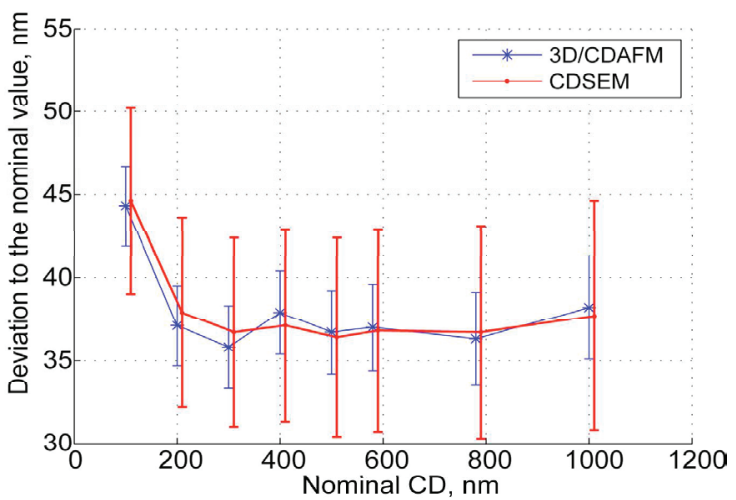

Fig.11Linewidth (CD) measurement results for isolated opaque line features on a photomask standard with estimated (preliminary) measurement uncertainties U95\%. All values in $\mathrm{nm}$.

\section{Nanoscale standards}

Besides sophisticated metrological tools, nanoscale standards also play an important role in realising reference nanometrology. Well calibrated high quality standards or reference materials are essential for, for instance, calibrating the probe geometry, testing or benchmarking tools, performing comparison measurements between different methods/tools as well as verifying theoretical modelling with experimental results.

A set of nanoscale standards have been successfully developed in the past two decades, for instance, step height and depth setting standards for calibrating the z-axis, 1D/2D gratings for calibrating the magnification and distortion of the xy plane, as well as the flatness standards for characterising the out-ofxy-plan motion of almost all kinds of microscopes. Recently also a new kind of 3D calibration standard with 3D-landmarks has been developed. In contrast to the commonly used calibration procedures which require separate lateral and height standards, the 3Dcalibration makes use of spatially distributed landmarks, i.e. special unique features defined by 3D-coordinates [22]. After the standard being calibrated by e.g. the metrological AFM, it is able to calibrate various tools such as optical microscopes (including confocal and phase shift microscopes), SEM and AFM by correlating the reference data sets and the data sets of the tool under calibration with just a single measurement.

In the recent years we have focused on the development of a new kind CD reference material collaborated with the Team nanotec $\mathrm{GmbH}$, as such reference material is inadequate for industry. The layout of the newly developed reference material referred to as IVPS100-PTB is shown in figure 11. The sample has a size of $6 \mathrm{~mm} \times 6 \mathrm{~mm}$, consisting of 4 groups of $5 \times 5$ feature patterns.
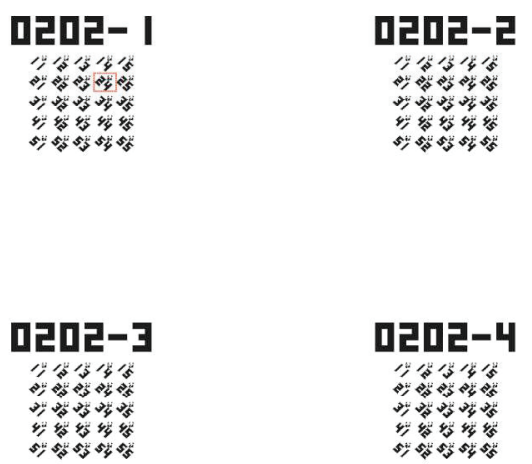

(a)

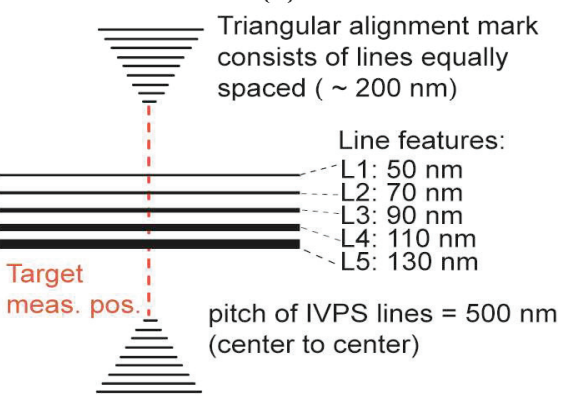

(b)

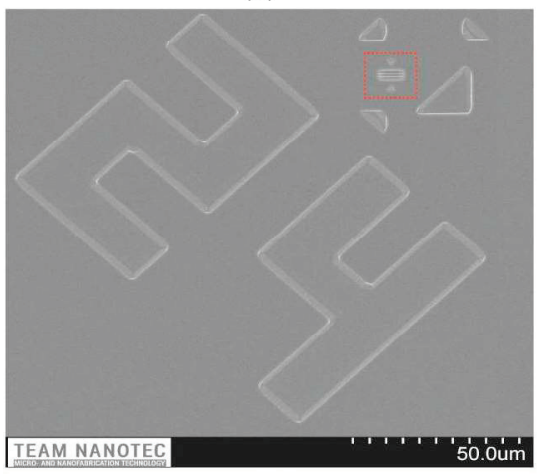

(c)

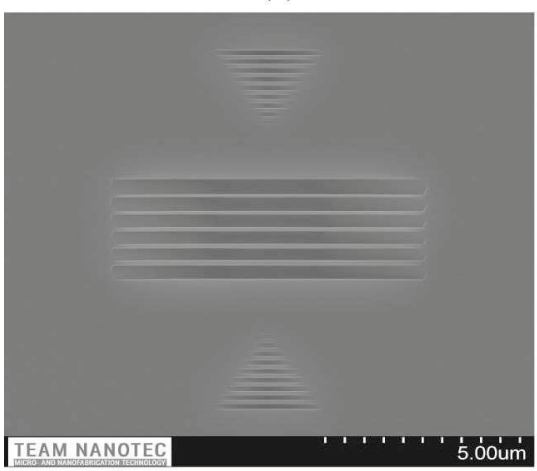

(d)

Fig.11. (a) Designed Layout of the sample consisting of 4 groups of feature patterns. Each pattern has 5 by 5 feature groups; (b) designed layout of a feature group with two alignment marks 
and a group of five line features; (c) SEM image of a fabricated feature group and its labeling numbers; (d) SEM image of the alignment marks and line features at the marked area in (c).

Each feature pattern has a group of five reference line features having vertical sidewalls with nominal CD of $50 \mathrm{~nm}, 70 \mathrm{~nm}, 90$ $\mathrm{nm}, 110 \mathrm{~nm}$ and $130 \mathrm{~nm}$, respectively. Each feature pattern includes a pair of triangular alignment marks, applicable for precisely identifying the target measurement position, e.g. for comparison or calibration between different tools.

The geometry of line features has been investigated thoroughly using a high resolution transmission electron microscope (HR-TEM) and our 3D/CD-AFM. Their results indicate the high quality of the line features: the top corner radius of $<7 \mathrm{~nm}$, vertical sidewall (slope mostly within $90^{\circ} \pm 0.5^{\circ}$ ), and very small line width variation (LWR down to $0.36 \mathrm{~nm}$ ) [23].

The developed reference material can be applied to calibrate probe geometry, test or benchmark CD tools, perform comparison measurements between different methods/tools as well as verify theoretical modelling with experimental results. The application of the sample for CD-AFM tip characterisation is given in figure 12 as an example. An IVPS-100-PTB is measured by a new CDR120-EBD tip (Nanosensor) which has a nominal tip width of $120 \mathrm{~nm}$. The obtained apparent CD-AFM values (i.e. the $C D$ values without tip correction) are plotted as the $y$ coordinates. After the CD-AFM measurements are performed, the sample is destructively prepared and TEM measured. The obtained $C D$ values of five line features by TEM are plotted as the $x$ coordinates.

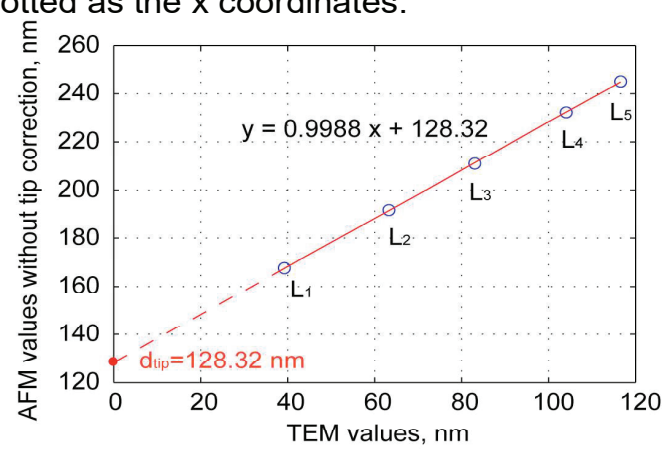

Fig.12.Calibration of the effective AFM tip width using the IVPS100-PTB standard. Five data points marked by circles indicate five pairs of TEM results and AFM results (raw data without tip correction) obtained on five line features, respectively. The red line is the leastsquare-fitted linear curve of the data points. The $y$-coordinate of the fitted curve at $x=0$ indicates the effective width of the applied AFM tip.

Fitting the obtained five data points to a linear function $\mathrm{y}=k \mathrm{x}+b$, we get:

$$
y=0.9988 x+128.32 n m
$$

Here the parameter $k=0.9988$ stands for the scaling factor of our CD-AFM. It coincides well with the theoretical value 1 , since the CD-AFM was calibrated to our metrological AFM in advance. The parameter $b=128.32 \mathrm{~nm}$ is the calibrated effective width of the applied tip.

For more info about the new reference materials, the readers are referred to [23]

\section{Conclusion}

Addressing the challenging metrology demands from nanotechnology related research and industries, reference nanometrology based on AFM, SEM and TEM technologies has been developed at PTB. It aims to provide accurate and traceable $3 D$ nano dimensional metrology services and standards for, for instance, calibrating, testing and benchmarking tools, performing comparison measurements between different methods/tools as well as verifying theoretical modelling with experimental results.

Several recent research progresses have been highlighted in the paper. To overcome the limit of low measurement speed of AFMs, a high speed Met. LR-AFM has been developed. It is capable of measuring with speed up to $1 \mathrm{~mm} / \mathrm{s}$ (i.e. 50x faster than before), thus significantly enhanced its measurement throughput and reduced the measurement drift. To enhance the true 3D measurement capability of the AFM technique, a 3D/CD-AFM which measures either with flared AFM tips or in the tilting-AFM principle, has been developed. It offers measurement capabilities for, e.g., CD, SWA, LER/LWR, corner rounding and footing of nanostructures. To address challenging issue in characterising the effect tip width for CD metrology, a new bottom-up traceability approach which applies the ${ }^{28} \mathrm{Si}$ crystal lattice as internal "rulers". By utilising the state-of-theart TEM tools which has capable spatial resolution down to $0.05 \mathrm{~nm}$, such reference metrology based on TEM can achieve sub-nm accuracy. Today, SEM is kind of fast and high resolution (1 $\mathrm{nm}$ ) measurement tool widely applied in nanomanufacturing for process development and process control, to ensure their measurement reliability, a metrological CD-SEM referred to as Electron-Optical Metrology System has been updated at PTB with a low-voltage SEM of the Zeiss ULTRA type. In order to properly interpret the yield 